\title{
Fichas clínicas simuladas en kinesiología
}

\author{
Simulated clinical records in kinesiology
}

Felipe Contreras-Briceño1', Cynthia Rojas-Muñoz', Jaime Passalacqua', Patricio García', Maximiliano Espinosa-Ramírez' , Antonio López-Fuenzalida' ${ }^{1}$, Javiera Fuentes ${ }^{1}$, Luigi Gabrielli Nervi ${ }^{2}$

\section{Resumen}

Introducción: la normativa actual de docencia clínica regula el desarrollo de actividades curriculares disminuyendo el tiempo de contacto estudiante-paciente, requiriendo entre otras destrezas del estudiante la lectura eficiente de información clínica. La simulación permite desarrollar competencias clínicas en los estudiantes de ciencias de la salud. El objetivo de este estudio es describir la experiencia de talleres de fichas clínicas simuladas (FCS) en estudiantes curriculares y reportar indicadores de logro de objetivos de aprendizaje relacionados al reconocimiento de las partes de la ficha clínica e identificación y extracción de información relevante. Metodología: en una asignatura de carácter mínimo previo al encuentro de estudiantes con pacientes reales, se desarrollaron cuatro talleres de FCS en grupo pequeño. Un académico guió la actividad consistente en responder cuestionarios de ubicación de información presente en las FCS. Al finalizar la asignatura, se evaluó la percepción de la didáctica educativa y logro de objetivos de aprendizaje en tutores clínicos y estudiantes mediante encuesta. Resultados: los estudiantes reportaron alta satisfacción con la metodología, facilidad para extraer información relevante y mayor tiempo de contacto clínico con pacientes reales. Los tutores clínicos informaron que los estudiantes logran reconocer las partes de la ficha clínica. Ambos consideran que el tiempo ideal para lectura de ficha clínica es de 10 a 20 minutos. Conclusión: la incorporación de talleres de fichas clínicas simuladas desarrolló habilidades clínicas de reconocimiento de las partes que componen la ficha clínica, optimizando el tiempo necesario para identificar y extraer información relevante a diferentes escenarios clínicos.

Palabras clave: simulación; razonamiento clínico; metodología educativa; aprendizaje; actividad clínica; comunicación.

\begin{abstract}
Introduction: The actual clinical teaching normative regulates the development of curricular activities, decreasing the student-patient contact time, making necessary a more efficient reading of the clinical information by the students. Simulation allows developing clinical skills in health professionals. This study describes the experience of simulated clinical record (SCR) workshops in undergraduate students and to report indicators of achievement of learning objectives on recognizing the parts of the clinical record and identify and extract relevant clinical information. Methodology: Four workshops of SCR were developed before the clinical visits in a subject in which students have the first clinical experience with real patients. The activity was guided by an academic, and it consisted of answering questionnaires of the location of information present in the SCR. At the end of the subject, clinical tutors and students evaluate the perception of these educational didactic and achievement of learning objectives using a dedicated survey. Results: Students reported high satisfaction with this methodology, recognizing that facilitates the learning process for obtaining relevant clinical information before the interview with real patients. On the other hand, the tutors reported that the students who performed the activity were trained to identify the parts of the clinical record. Both consider that the time-optimal dedicated to reading the clinical history is 10 to 20 minutes. Conclusion: Among students, the simulated clinical records workshops allowed the development of clinical skills related to identify, recognize, and optimize the time necessary to extract relevant clinical information in different clinical scenarios.
\end{abstract}

Keywords: Simulation; Clinical reasoning; Educational methodology; Learning; Clinical activity; Communication.

Fecha de envío: 29 de julio de 2019 - Fecha de aceptación: 12 de diciembre de 2019

(1) Departamento Ciencias de la Salud, Facultad de Medicina, Pontificia Universidad Católica de Chile.

(2) División de Enfermedades cardiovasculares, Facultad de Medicina, Pontificia Universidad Católica de Chile.

Autor correspondencia: fcontrerasb@uc.cl 


\section{Introducción}

La modificación del marco regulatorio de la relación asistencial docente entre carreras de la salud y campos clínicos en Chile (Ministerio de Salud, 2017) a propósito de la nueva Ley de Derechos y Deberes de los Pacientes (Ministerio de Salud, 2012) , ha implicado en la dinámica habitual de docencia clínica tutorial realizada por profesionales con labor asistencial, una disminución del tiempo destinado a demostrar con el paciente real las habilidades clínicas a adquirir por parte de los estudiantes, aminorando el tiempo de contacto que éstos tienen con los pacientes, afectando así el proceso de razonamiento clínico, autorreflexión, y control del aprendizaje propio de los estudiantes. Esta situación motiva la incorporación de nuevas didácticas educativas orientadas en la innovación en asignaturas clínicas propias de las diferentes mallas curriculares de estas carreras, con la finalidad de favorecer el logro de objetivos de aprendizaje que permitan la adquisición de competencias profesionales relacionadas al ámbito clínico (Villagrán et al., 2018). En este contexto, la simulación clínica definida como la reproducción de situaciones o procedimientos con el fin de entrenar competencias clínicas en ambientes seguros, ha demostrado promover el aprendizaje a través de una retroalimentación inmediata y oportuna, desarrollando competencias clínicas de manera previa al enfrentamiento del escenario clínico real, permitiendo el logro de objetivos de aprendizaje a través del ensayo y error en ambientes protegidos desde el punto de vista educativo, siendo una herramienta útil para la resolución de problemáticas educacionales (Escudero et al., 2016). En estudiantes de tercer año de enfermería, la incorporación de una metodología de entrenamiento de simulación perinatal ha reportado un aumento de las competencias y confianza después del entrenamiento, destacando que la metodología les permite confrontar sus propias posibilidades de rendimiento y conocimiento (Vermeulen et al., 2017). Lo mismo fue reportado en residentes de medicina interna y urgencias, donde una intervención educativa basada en la simulación de habilidades de inserción de catéter venoso central (CVC), redujo significativamente la incidencia de infección asociada a dicho procedimiento, destacando la educación basada en simulación como un pilar fundamental en la capacitación clínica estándar (Barsuk et al., 2015).

El manejo de la información contenida en las fichas clínicas es una competencia que debe ser desarrollada por todos los profesionales de la salud, pues se ha documentado que un adecuado manejo y almacenamiento de la información contenida en las fichas clínicas impacta directamente en la salud de los pacientes (Wald et al., 2014). La World Confederation for Physycal Therapy (WCPT) ha definido estándares que explicitan que los programas de formación deben contener instancias de aprendizaje en su planificación que intencionen el manejo, cuidado y entrega de información contenida en las fichas clínicas (World Confederation for Physycal Therapy, 2011).
Considerando este escenario, en la carrera de kinesiología UC los estudiantes de tercer año comienzan con actividades clínicas en los diferentes centros en convenio. Así, la asignatura de semiología y anamnesis para kinesiología impartida en quinto semestre de la malla curricular es un curso de carácter mínimo u obligatorio y constituye el primer acercamiento clínico de los estudiantes con pacientes reales, teniendo los siguientes objetivos de aprendizaje: (i) reconocer las partes que componen la ficha clínica; (ii) identificar y extraer información relevante de los diferentes contextos clínicos; (iii) favorecer el desarrollo de habilidades clínicas ligadas al tiempo de contacto del estudiante con pacientes reales. En este contexto, una de las dificultades observadas en los estudiantes es el tiempo prolongado que implica la lectura de ficha clínica y la dificultad para identificar y extraer información relevante para cada contexto clínico durante las experiencias curriculares con pacientes reales (visitas clínicas), actividades frecuentes en las asignaturas del plan de estudios de todas las carreras en ciencias de la salud, dificultando así la adquisión y desarrollo de las habilidades clínicas propias de la disciplina. Dado este escenario, surge la necesidad de incorporar didácticas educativas en la formación en kinesiología que faciliten el desarrollo de habilidades clínicas relacionadas con el reconocimiento de las partes de la ficha clínica, y la identificación y extracción adecuada de información relevante a cada escenario, empleando así el tiempo de lectura de forma más eficiente y con ello favorecer un mayor y mejor tiempo de contacto de los estudiantes con los pacientes reales en las actividades prácticas en los centros clínicos a través de un entrenamiento en la lectura de fichas clínicas.

Por lo tanto, el objetivo de este estudio es describir la experiencia de la incorporación de talleres de fichas clínicas simuladas en estudiantes de kinesiología UC y reportar los indicadores de logro de objetivos de aprendizaje orientados en reconocer las partes que componen la ficha clínica e identificar y extraer información relevante a cada escenario clínico.

\section{Metodología}

El diseño de estudio es descriptivo con análisis retrospectivo. Con la finalidad de robustecer los resultados con comentarios relevantes, se utilizó la metodología de análisis de contenidos de las encuestas de los participantes (estudiantes y tutores clínicos).

\section{Fichas clínicas}

Se utilizó un total de 6 escenarios clínicos reales englobando el área médica y enfermería, reemplazando los datos personales de pacientes reales por información ficticia. Estas fichas abarcaron diferentes áreas de desarrollo profesional para la kinesiología, involucrando temáticas del área musculoesquelética, neurológica y cardiorrespiratoria, e incluyendo abreviaturas, acrónimos, 
diagnósticos, y exámenes comunes a la clínica habitual. Todas las fichas clínicas fueron evaluadas y aprobadas por un comité de expertos de cada área, quienes mejoraron los contenidos más comunes que el estudiante debiese conocer.

\section{Didáctica educativa}

La metodología docente del proyecto fue impartida en la asignatura de semiología y anamnesis para kinesiología. Esta asignatura de carácter mínimo u obligatoria se desarrolla en el quinto semestre de la malla curricular, y constituye el primer acercamiento clínico de los estudiantes con pacientes reales.

La didáctica se realizó como trabajo en grupos pequeños de 2 a 3 estudiantes en cuatro talleres de tres horas de duración cada uno durante el semestre, los cuales fueron calendarizados de forma previa a las visitas clínicas con pacientes reales. El primer taller consistió en una clase expositiva-participativa cuyo objetivo fue que los estudiantes conocieran la estructura de la ficha clínica y comprendieran su relevancia como instrumento médico legal. Fue realizada por un médico y abogado expertos en el área. En los siguientes tres talleres, los grupos pequeños pudieron trabajar en las actividades propias de la didáctica consistentes en reconocer las partes de la ficha clínica; e identificar y extraer la información relevante de cada contexto clínico. Así, un académico participante en la asignatura trabajó con cinco grupos pequeños de estudiantes, y se encargó de guiar y solucionar inquietudes relacionadas al contexto clínico de cada escenario presente en las diferentes fichas clínicas. Cada grupo contó con una hora y treinta minutos para dar respuesta a preguntas abiertas relacionadas a la temporalidad y acciones clínicas que se mencionaban en el transcurso de la historia del paciente. En cada uno de los talleres, cada grupo pequeño trabajó dos fichas clínicas de diferentes áreas distribuidas de forma aleatoria. Al finalizar cada uno de los talleres, el académico entregaba las respuestas correctas de los diferentes cuestionarios propios de cada ficha con la finalidad de que los estudiantes reconocieran el porcentaje de logros de objetivos de la actividad, comparando sus respuestas con la pauta modelo, identificando dónde se encontraba la información solicitada dentro de la ficha clínica. Al final de estas didácticas los estudiantes pudieron desarrollar cuestionarios de cada una de las seis fichas clínicas.

\section{Evaluación de la intervención educacional}

Al término de la asignatura se invitó mediante correo electrónico y enlace web tanto a estudiantes de la asignatura como a los tutores clínicos que los guiaron durante las cuatro visitas clínicas con pacientes reales, a responder un cuestionario compuesto por preguntas relacionadas al logro de los objetivos de aprendizaje de la asignatura consistentes en reconocer las partes que componen la ficha clínica, identificar y extraer información relevante de los diferentes contextos de las visitas con pacientes reales, y favorecer el desarrollo de habilidades clínicas ligadas al tiempo de contacto del estudiante con los pacientes (comunicación efectiva en contexto de entrevista clínica).

Se diseñaron dos encuestas con un total de ocho preguntas para los estudiantes y seis para los tutores clínicos. Las respuestas se almacenaron en formato digital en plataforma SurveyMonkey ${ }^{\circledR}$, y eran de carácter anónimo. El plazo máximo de respuesta para el cuestionario fue de veintiocho días. El diseño del estudio se presenta en la Figura 1.

\section{Población}

El número de estudiantes que realizó la metodología de fichas clínicas simuladas fue $246 ; 28 \%(n=70)$ correspondientes a la cohorte 2016, 41\% $(n=101)$ a cohorte 2017 y $31 \%(n=75)$ a cohorte 2018. Participaron además 6 académicos de la asignatura que guiaron los talleres de fichas clínicas simuladas y 22 tutores clínicos encargados de guiar las actividades clínicas con pacientes reales.

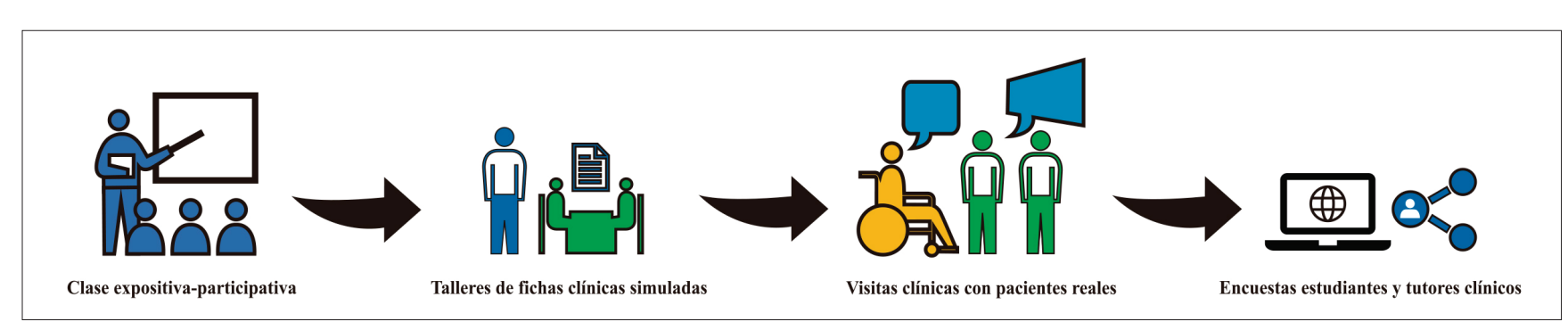

Figura 1: Metodología del estudio. Secuencia de actividades desarrolladas durante la implementación de la didáctica educativa de fichas clínicas simuladas.

\section{Resultados}

Las respuestas a cada una de las preguntas de los cuestionarios se presentan como número de casos y porcentaje del total.
Para valorar la percepción de los encuestados, se presentan además las expresiones y/o frases más reiterativas citadas de forma literal por los encuestados. 
De los 246 estudiantes participantes de la didáctica educativa, 171 (70\%) respondieron la encuesta enviada al finalizar la asignatura. De ellos, 35,09\% ( $n=60)$ correspondían a la cohorte $2016,45,03 \%$ $(n=77)$ a la cohorte 2017 y $19,88 \%(n=34)$ a la cohorte 2018 . Por otra parte la totalidad de tutores clínicos invitados a participar $(n=22)$ respondieron la encuesta.

Los resultados se presentan en base a los ítems que involucraba la encuesta:

- ítem 1 grado de satisfacción con la metodología

- ítem 2 reconocimiento de las partes de la ficha clínica

- ítem 3 identificación y extracción de información relevante

- ítem 4 tiempo necesario para la lectura de ficha clínica

Las respuestas de los estudiantes incluyen los ítems 1, 3 y 4, mientras que las respuestas de los tutores clínicos incluyen los ítems 2, 3 y 4.

\section{Respuesta de los estudiantes (tabla 1)}

\section{Ítem 1. Grado de satisfacción}

Respecto al nivel de satisfacción de los estudiantes con la didáctica educativa, tres preguntas de la encuesta $(1,7,8)$ abarcaron este ítem. En la pregunta 1, el $94,15 \%$ de los estudiantes $(n=161)$ consideró que la metodología del proyecto fue útil, refiriendo que E1: "es útil que se puedan dar instancias para poder aprender a leer fichas clínicas" y E2: "es una actividad muy provechosa". Por otra parte, el $5,85 \%$ ( $n=10$ ) consideró que la metodología no fue útil, declarando que E1: "las fichas clínicas revisadas en clase deberían ser realmente extensas, como aquellas que nos toca revisar en las visitas" y E2: "es mejor que se lea la ficha individualmente ya que en parejas o tríos es más complicado".

Respecto a la pregunta 7, el 78,95\% de los estudiantes $(n=135)$ refiere que los talleres de fichas clínicas simuladas son E1: "una actividad excelente, ya que es lo más importante junto a la entrevista y evaluación para poder tratar al paciente de forma efectiva"; E2: "una actividad muy necesaria para nuestra formación"; E3: "una forma de vincularnos más al contexto clínico del paciente" y E4: "una metodología de trabajo que está muy conectada con el aprendizaje que se nos otorga". El resto de los encuestados refirió que E1: "me hubiese gustado extraer la información que a mí me parecía relevante más que la que se pedía en la actividad" y que E2: "realizar 4 talleres sobre lo mismo se me hizo tedioso".

Al ser consultados por la calificación (pregunta 8) para la metodología de fichas clínicas simuladas desarrollada en la asignatura en una escala de 1 a 7, el 69,01\% de los estudiantes ( $n=118)$ calificó la actividad en un rango entre 6 y $7,24,56 \%(n=42)$ entre 5 y $6,5,26 \%$ $(n=9)$ entre 4 y 5 , y $1,17 \%(n=2)$ entre 3 y 4 . No hubo comentarios respecto a esta pregunta.

\section{Ítem 3. Identificación y extracción de información relevante}

Este ítem fue evaluado a través de las preguntas 2, 3 y 4 . En la pregunta $2,74,27 \%$ de los estudiantes encuestados ( $n=127)$ refirió percibir mayor facilidad para extraer información de manera adecuada de las fichas clínicas en sus visitas con pacientes reales, mencionando que E1: "Ios talleres servían como introducción a conocer cómo se ordena una ficha y la posible información que podemos encontrar"; E2: "en todas las visitas logré junto a mis compañeros extraer la información relevante" y que E3: "de todas maneras el taller fue una ayuda". El 23,39\% ( $n=40)$ respondió que esta facilidad para extraer la información ocurrió algunas veces, declarando que E1: "en los campos clínicos las fichas clínicas eran en su mayoría extensas y no seguían un orden claro como el de las fichas simuladas"; E2: "Ias fichas de los hospitales eran muy desordenadas"; y que E3: "en algunos campos clínicos no entendíamos la letra escrita en las fichas clínicas".

Respecto a la pregunta 3 y la transferencia de lo aprendido a la práctica en las visitas clínicas reales, $86,55 \%(n=148)$ respondió que el haber participado de los talleres de fichas clínicas simuladas les permitió tener un mayor contacto clínico con los pacientes que entrevistaron declarando que E1: "ayudó para saber específicamente a qué información dirigirse en la entrevista del paciente para poder completar la historia clínica" y E2: "nos permitió tener más tiempo para entrevistar a nuestro paciente". El porcentaje restante de los encuestados declaró que E1: "no tuve contacto con pacientes en las visitas clínicas" y que E2: "no siempre tuve más tiempo con el paciente ya que en dos oportunidades entró personal para procedimientos y tuvimos que abandonar la habitación".

Por otra parte, respecto a los objetivos de las visitas clínicas (pregunta 4), el 91,81\% de los estudiantes ( $n=157)$ respondió que la metodología utilizada en la asignatura facilitó el cumplimiento de los objetivos planteados para las actividades en campo clínico, mencionando que E1: "los talleres me prepararon mucho para las visitas, de manera que al poder rescatar la información más relevante de los pacientes, pude guiarme y realizar una entrevista adecuada, teniendo súper claro la historia del entrevistado". El resto de los encuestados que respondió "no" o " a veces", detalló que E1: "no siempre sentí eso ya que al haber fichas muy largas y con letra que no se entendía, ocupé más tiempo en la lectura restando tiempo a la entrevista, y muchas veces no alcanzaba a preguntar todo lo que quería"; E2: "al leer una ficha muy larga, no sabía bien en qué enfocarme para hacer la entrevista, pero creo que es solo falta de experiencia y que mejorará con más práctica en otros cursos".

\section{Ítem 4. Tiempo necesario para lectura de ficha clínica}

En relación al tiempo destinado a la lectura de fichas clínicas considerado en las preguntas 5 y $6,61,40 \%$ de los estudiantes $(n=105)$ declaró haber destinado entre 10 y 20 minutos del tiempo total de 
la visita a la lectura de ficha clínica. Y al ser consultados respecto al tiempo necesario para comprender adecuadamente la historia clínica de un paciente a través de la información que aparece en las fichas clínicas, $42,69 \%$ ( $n=73$ ) respondió que deberían contemplarse hasta 10 minutos y $34,50 \%$ ( $n=59)$ declaró que debería considerarse un rango entre 10 y 20 minutos para comprender adecuadamente la historia clínica de un paciente real. No hubo comentarios por parte de los estudiantes encuestados para las preguntas 5 o 6 .

Tabla 1: Resultados de la encuesta de los estudiantes ( $n=171)$.

\begin{tabular}{|c|c|c|c|c|}
\hline $\mathbf{N}^{\circ}$ & Pregunta & Respuesta & $\mathbf{N}^{\circ}$ de casos & Porcentaje (\%) \\
\hline \multirow{2}{*}{1} & \multirow{2}{*}{ ¿Considera que la metodología docente fue útil para el objetivo del proyecto? } & $\mathrm{Si}$ & 161 & 94,1 \\
\hline & & No & 10 & 5,85 \\
\hline \multirow{3}{*}{2} & \multirow{3}{*}{$\begin{array}{l}\text { En las visitas clínicas que realizó en la asignatura, ¿considera que la actividad } \\
\text { de los talleres previos le permitió extraer de manera adecuada la información } \\
\text { relevante de las fichas clínicas? }\end{array}$} & Si & 127 & 74,27 \\
\hline & & No & 4 & 2,34 \\
\hline & & A veces & 40 & 23,39 \\
\hline \multirow{3}{*}{3} & \multirow{3}{*}{$\begin{array}{l}\text { ¿Considera Ud. que el haber participado los talleres previos de fichas clínicas } \\
\text { simuladas le permitió en las visitas clínicas reales tener un mayor contacto } \\
\text { clínico con los pacientes? }\end{array}$} & Si & 148 & 86,55 \\
\hline & & No & 10 & 5,85 \\
\hline & & A veces & 13 & 7,60 \\
\hline \multirow{3}{*}{4} & \multirow{3}{*}{$\begin{array}{l}\text { ¿Considera Ud. que el manejo de la ficha clínica reales demostrado en las } \\
\text { visitas clínicas les facilitó el cumplimiento de los objetivos de estas? }\end{array}$} & $\mathrm{Si}$ & 157 & 91,81 \\
\hline & & No & 3 & 1,75 \\
\hline & & A veces & 11 & 6,43 \\
\hline \multirow{4}{*}{5} & \multirow{4}{*}{$\begin{array}{l}\text { ¿Cuánto tiempo considera Ud. le destinó al uso de la ficha clínica reales en las } \\
\text { visitas clínicas para lograr extraer la información necesaria y útil? }\end{array}$} & Hasta 10 minutos & 15 & 8,77 \\
\hline & & 10 a 20 minutos & 105 & 61,40 \\
\hline & & 20 a 30 minutos & 45 & 26,32 \\
\hline & & Más de 30 minutos & 5 & 2,92 \\
\hline \multirow{4}{*}{6} & \multirow{4}{*}{$\begin{array}{l}\text { ¿Cuánto tiempo considera Ud. se debería otorgar en la realidad para } \\
\text { comprender adecuadamente la historia clínica de un paciente a través de la } \\
\text { información que aparece en las fichas clínicas reales? }\end{array}$} & Hasta 10 minutos & 73 & 42,69 \\
\hline & & 10 a 20 minutos & 59 & 34,50 \\
\hline & & 20 a 30 minutos & 33 & 19,30 \\
\hline & & Más de 30 minutos & 6 & 3,51 \\
\hline \multirow{5}{*}{7} & \multirow{5}{*}{ La actividad de los talleres de fichas clínicas simuladas la encuentra: } & $\begin{array}{l}\text { Una experiencia muy } \\
\text { enriquecedora en mi } \\
\text { formación para cursos } \\
\text { superiores }\end{array}$ & 135 & 78,95 \\
\hline & & $\begin{array}{l}\text { Adecuada pero no ne- } \\
\text { cesaria para desarrollar } \\
\text { una buena visita clínica }\end{array}$ & 15 & 8,77 \\
\hline & & $\begin{array}{l}\text { Prescindible para las } \\
\text { visitas clínicas }\end{array}$ & 10 & 5,85 \\
\hline & & $\begin{array}{l}\text { Una pérdida de tiempo, } \\
\text { considerando las demás } \\
\text { actividades que se } \\
\text { podrían desarrollar en la } \\
\text { asignatura }\end{array}$ & 3 & 1,75 \\
\hline & & $\begin{array}{c}\text { Muy mal organizadas, } \\
\text { los docentes no ayuda- } \\
\text { ron en nada el desarrollo } \\
\text { de la actividad }\end{array}$ & 0 & 0.00 \\
\hline \multirow{5}{*}{8} & \multirow{5}{*}{$\begin{array}{l}\text { ¿Qué nota le pondría a la actividad de talleres de fichas clínicas simuladas? } \\
\text { (escala } 1 \text { a 7) }\end{array}$} & Entre 1 y 3 & 0 & 0,00 \\
\hline & & 3 y 4 & 2 & 1,17 \\
\hline & & 4 y 5 & 9 & 5,26 \\
\hline & & 5 y 6 & 42 & 24,56 \\
\hline & & 6 y 7 & 118 & 69,01 \\
\hline
\end{tabular}




\section{Respuesta de los tutores clínicos (tabla 2)}

\section{Ítem 2. Identificación de las partes de la ficha clínica}

Este ítem fue evaluado en la pregunta 1 de la encuesta realizada a los tutores clínicos, donde el 90,91\% de los encuestados $(n=20)$ consideró que los estudiantes fueron capaces de identificar las partes que componen una ficha clínica, y 9,09\% $(n=2)$ respondió "a veces" detallando que T1: "en algunas oportunidades los estudiantes no lograban identificar dónde encontrar las indicaciones del día" y T2: "en el caso de fichas muy extensas, perdían parte de la evolución del paciente".

\section{Ítem 3. Identificación y extracción de información relevante}

La identificación y extracción de información relevante de la ficha clínica fue incluida en las preguntas 2, 3 y 4 . Para la pregunta 2, 63,64\% $(n=14)$ respondió que los estudiantes fueron capaces de extraer información relevante de las fichas clínicas, detallando que T1: "los estudiantes lograron extraer adecuadamente la información relevante de las fichas clínicas la mayor parte de las veces. Identificaron motivos de consulta, diagnóstico, plan médico, entre otros."T2: "los estudiantes manejaban muy bien las partes que conformaban la ficha clínica y en la última visita su lectura fue mucho más fluida y la extracción de la información mucho más precisa y atingente". Aquellos tutores clínicos que respondieron "a veces" detallaron que T1: "los estudiantes son capaces de extraer la información, pero dado su nivel, como no conocen muchos conceptos aún, pierden seguridad en la búsqueda" y T2: "en algunas ocasiones, especialmente al leer fichas extensas, los estudiantes presentaban dificultad para rescatar hitos relevantes de la evolución".

Respecto a la pregunta 3,72,73\% de los tutores encuestados ( $n=16$ ) respondió que el manejo de los estudiantes con la ficha les permitió un mayor contacto clínico con los pacientes, T1: "especialmente en las últimas visitas, donde ocupaban menor tiempo en leery más tiempo en la entrevista". El resto de los tutores clínicos que respondió "a veces" o "no", detalló que el tiempo de contacto clínico T1: "depende de la motivación personal del estudiante"; T2: "se ve afectado por la poca experiencia con pacientes reales que tiene los estudiantes en el sexto semestre de la malla curricular" y que T3: "es difícil establecer un cambio o comparar si no conocí a los estudiantes previo a la metodología".

En relación a la pregunta 4, 90,91\% $(n=20)$ considera que el manejo de ficha clínica facilitó el cumplimiento de los objetivos de las visitas clínicas con pacientes reales, declarando que T1: "les facilita comprender la historia del paciente y los motiva a indagar más en la entrevista". 4,55\% ( $n=1)$ contestó que esto ocurrió "a veces".

\section{Ítem 4. Tiempo necesario para lectura de ficha clínica}

El tiempo destinado a la lectura de fichas clínicas fue considerado en las preguntas 5 y 6 . Para la pregunta 5, 54,55\% de los tutores clínicos $(n=12)$ respondió que los estudiantes destinaron entre 20 y 30 minutos del tiempo total de la visita a la lectura de ficha clínica, $31,82 \%(n=7)$ respondió 10 a 20 minutos, 9,09\% $(n=2)$ más de 30 minutos y $4,55 \%(n=1)$ hasta 10 minutos. Al ser consultados respecto al tiempo necesario para comprender adecuadamente la historia clínica de un paciente a través de la información que aparece en las fichas clínicas (pregunta 6), 54,55\% de los tutores ( $n=12$ ) indicó que debería considerarse un margen de tiempo entre 10 y 20 minutos, $22,73 \%(n=5)$ respondió hasta 10 minutos, $13,64 \%(n=3)$ entre 20 y 30 minutos y $4,55 \%(n=1)$ más de 30 minutos. No hubo comentarios por parte de los tutores clínicos encuestados para las preguntas 5 o 6.

Tabla 2: Resultados de la encuesta de los tutores clínicos ( $n=22)$.

\begin{tabular}{|c|c|c|c|c|}
\hline $\mathbf{N}^{\circ}$ & Pregunta & Respuesta & $\mathbf{N}^{\circ}$ de casos & Porcentaje (\%) \\
\hline \multirow{3}{*}{1} & \multirow{3}{*}{$\begin{array}{l}\text { ¿Considera Ud. que los alumnos fueron capaces de identificar las partes que } \\
\text { componen una ficha clínica? (ej.: ingreso a urgencia, evolución clínica, exámenes, } \\
\text { registro de signos vitales, etc) }\end{array}$} & $\mathrm{Si}$ & 20 & 90,91 \\
\hline & & No & 0 & 0,00 \\
\hline & & A veces & 2 & 9,09 \\
\hline \multirow{3}{*}{2} & \multirow{3}{*}{$\begin{array}{l}\text { ¿Fueron capaces de extraer de manera adecuada la información relevante de las } \\
\text { fichas clínicas durante las visitas donde Ud. participó como tutor? }\end{array}$} & $\mathrm{Si}$ & 14 & 63,64 \\
\hline & & No & 0 & 0,00 \\
\hline & & A veces & 8 & 36,36 \\
\hline \multirow{3}{*}{3} & \multirow{3}{*}{$\begin{array}{l}\text { ¿Considera Ud. que el manejo presentado de la ficha clínica les permitió tener un } \\
\text { mayor contacto clínico con los pacientes (ej. desarrollo de preguntas abiertas, etc.)? }\end{array}$} & $\mathrm{Si}$ & 16 & 72,73 \\
\hline & & No & 1 & 4,55 \\
\hline & & A veces & 5 & 22,73 \\
\hline \multirow{3}{*}{4} & \multirow{3}{*}{$\begin{array}{l}\text { ¿Considera Ud. que el manejo de la ficha clínica demostrado por los alumnos les } \\
\text { facilitó el cumplimiento de los objetivos de las visitas clínicas? }\end{array}$} & $\mathrm{Si}$ & 20 & 90,91 \\
\hline & & No & 0 & 0,00 \\
\hline & & A veces & 1 & 4,55 \\
\hline \multirow{4}{*}{5} & \multirow{4}{*}{$\begin{array}{l}\text { ¿Cuánto tiempo considera Ud. que los alumnos destinaron al uso de la ficha clínica } \\
\text { para extraer la información necesaria para desarrollar la visita clínica? }\end{array}$} & Hasta 10 minutos & 1 & 4,55 \\
\hline & & 10 a 20 minutos & 7 & 31,82 \\
\hline & & 20 a 30 minutos & 12 & 54,55 \\
\hline & & Más de 30 minutos & 2 & 9,09 \\
\hline \multirow{4}{*}{6} & \multirow{4}{*}{$\begin{array}{l}\text { ¿Cuánto tiempo considera Ud. es necesario para comprender adecuadamente } \\
\text { la historia clínica de un paciente a través de la información que aparece en las } \\
\text { fichas clínicas? }\end{array}$} & Hasta 10 minutos & 5 & 22,73 \\
\hline & & 10 a 20 minutos & 12 & 54,55 \\
\hline & & 20 a 30 minutos & 3 & 13,64 \\
\hline & & Más de 30 minutos & 1 & 4,55 \\
\hline
\end{tabular}




\section{Discusión}

La incorporación de talleres de simulación en fichas clínicas en estudiantes de kinesiología permitió el desarrollo de competencias relacionadas al reconocimiento de las partes que componen el documento estándar de ficha clínica, favoreciendo la identificación y extracción adecuada de información relevante a diferentes escenarios clínicos.

En el contexto de la Ley de Derechos y Deberes de los pacientes (Ministerio de Salud, 2012) y los cambios en el marco regulatorio de las visitas clínicas de estudiantes de pregrado que ha provocado disminución en el tiempo de contacto clínico con pacientes reales dado el marco regulatorio relacionado a privacidad y procedimientos técnicos inocuos factibles de realizar por estudiantes en formación, la simulación ha demostrado ser una metodología atractiva y eficaz para el proceso de aprendizaje, constituyendo un método de enseñanza idóneo para el desarrollo de competencias clínicas que contribuyan al logro de objetivos de aprendizaje (Utili, 2007).

Similar a otros estudios, el análisis de la encuesta del presente estudio muestra que los estudiantes valoran los talleres de simulación con una elevada satisfacción y calificación, lo que evidencia una vez más que el uso de la simulación produce una alta conformidad, mayor conocimiento y mejor desempeño por parte de los estudiantes (Weller et al., 2012). Por lo tanto, este estudio permite considerar el uso de fichas clínicas simuladas como una metodología útil para el desarrollo de una lectura eficiente y eficaz en campos clínicos reales y que proyecta una posible relación entre la percepción de lo aprendido y la experiencia clínica del estudiante (Villagrán et al., 2018). Si bien hasta la fecha no se cuenta con información respecto a estudios similares que utilicen esta didáctica educativa, otros artículos han destacado la práctica deliberada como una propiedad importante dentro de la simulación clínica que permite desarrollar y mantener además del conocimiento, habilidades y actitudes en los estudiantes, tal como queda demostrado en el presente estudio (McGaghie et al., 2010).

Los estudiantes encuestados perciben un cambio favorable en el rescate de información relevante en las fichas clínicas, lo cual es uno de los principales objetivos de este estudio y coincide con lo manifestado por los tutores clínicos. Sin embargo, parte de los estudiantes encuestados refieren haber percibido dicho cambio solo en algunas ocasiones (23,39\%). Una posible explicación para ello es la presencia de fichas clínicas muy extensas y escritas en letra manuscrita de difícil lectura y comprensión, de forma que el reconocimiento de información se limita, aspecto que también es referido por los tutores clínicos. En este contexto, algunos estudiantes declararon que E1: "si bien me permitió extraer mucha información relevante, en fichas clínicas más extensas donde la información es mayor, no me sentí capaz de extraer lo más útil y relevante". Además, agregan respecto al formato de las fichas clínicas en campos clínicos que E2: "las fichas son diferentes en todos los centros de visita clínica por lo que a veces se dificulta extraer información", y E3: "muchas veces los formatos son distintos dependiendo del centro clínico, pero en general la metodología de fichas clínicas simuladas si ayuda".

Por otro lado, los estudiantes refieren que el haber realizado una lectura más eficiente de la ficha clínica gracias a la metodología impartida en la asignatura, les permitió tener un mayor tiempo de contacto clínico con sus pacientes, lo cual facilitó el cumplimiento de los objetivos de las actividades clínicas en contexto real, aspecto que es concordante con lo referido por los tutores de campo clínico y deja en evidencia al igual que la literatura revisada que la simulación promueve el aprendizaje basado en la experiencia (Escudero et al., 2016). Esto es especialmente importante, ya que una adecuada lectura e interpretación de la historia clínica del paciente permite un mayor tiempo para realizar evaluación y tratamiento de pacientes, aspectos que precisamente se abarcan y son el objetivo principal en asignaturas de cursos superiores dentro de la malla curricular de kinesiología. En base a este antecedente, la metodología docente utilizada en este estudio podría ser replicada en cualquier carrera del área de la salud que desarrolle actividades prácticas con pacientes reales donde el estudiante deba extraer información a partir de fichas clínicas, como es el caso de medicina, odontología, nutrición, fonoaudiología, entre otras.

Un aspecto importante evaluado en este estudio es el tiempo que tanto estudiantes como tutores clínicos consideran debería ser entregado para una lectura de ficha clínica eficaz y la extracción de información relevante, el cual es menor o igual a 20 minutos (ver tablas 1 y 2). Esto es importante a considerar en cursos superiores, pues permitirá incorporarlo a las pautas de evaluación de exámenes clínicos. Actualmente en kinesiología UC el tiempo considerado a ese ítem dentro de los instrumentos de evaluación en asignaturas de tercer y cuarto año es de 10 minutos, requiriendo modificación a futuro y entrega de información de estos resultados a académicos y estudiantes, con el objetivo de que conozcan el origen del fundamento, y evaluar si dicho tiempo se mantiene o disminuye a medida que el número de visitas clínicas se incrementa.

Una de las limitaciones del presente estudio es que la encuesta aplicada a los estudiantes solo logró evaluar el nivel 1 (satisfacción) del "Modelo de Evaluación de Programas de Formación de Kirkpatrick", el cual es considerado el marco de referencia para determinar el impacto de programas de formación (Kirkpatrick, 1979; Kirkpatrick \& Kirkpatrick, 2012). Para poder evaluar niveles 
superiores y lograr determinar el impacto educacional de la presente intervención, es necesario desarrollar o contar con insumos que permitan determinar el aprendizaje, la transferencia de lo aprendido a la práctica clínica y eventualmente, el impacto en el paciente y/o en la organización. Además, la metodología educativa empleada en este estudio requiere evaluación a largo plazo, ya que no se tienen datos relevantes respecto a indicadores a largo plazo en relación al desarrollo de esta didáctica educativa.

En resumen, este estudio demostró que la didáctica educativa de fichas clínicas simuladas impartida en la asignatura de semiología y anamnesis para kinesiología, donde los estudiantes de quinto semestre experimentan el primer contacto clínico con pacientes reales, permite que los estudiantes reconozcan las partes que componen la ficha clínica y con ello poder identificar y extraer la información más relevante para un escenario clínico determinado. Esto va en directo beneficio a la formación de los estudiantes en cursos superiores, pues se espera que en las asignaturas propias de cada área de la kinesiología, puedan acercarse al paciente con seguridad y confianza gracias al entendimiento de manera temprana de la historia clínica, ya que una lectura eficiente y efectiva de la ficha clínica permitirá que los estudiantes puedan disponer de mayor tiempo para la evaluación y tratamiento de sus pacientes, facilitando la incorporación de habilidades clínicas propias de cada área y con ello, el cumplimiento de los objetivos de aprendizaje de asignaturas y visitas clínicas futuras.

\section{Agradecimientos}

Este estudio recibió apoyo financiero de Fondo de Desarrollo de la Docencia de la Pontificia Universidad Católica de Chile (FONDEDOC; Inv. Resp: (Revisión por pares anónima).

\section{Conflictos de interés}

Los autores declaran no presentar conflicto de interés.

\section{Contribuciones}

(Revisión por pares anónima)

\section{Referencias}

Barsuk J, Cohen E, Feinglass J, McGaghie W \& Wayne D. (2015). Use of Simulation-Based Education to Reduce Catheter-Related Bloodstream Infections. JAMA Internal Medicine 169, 1420-1423.

Escudero E, Fuentes C, González M \& Corvetto M. (2016). Simulación en educación para ciencias de la salud: ¿Qué calidad hemos alcanzado en Chile? Ars Medica Revista de Ciencias Médicas 2, 16-20.

Kirkpatrick D. (1979). Techniques for Evaluating Training Programs. Training And Development Journal, 78-92.
Kirkpatrick D \& Kirkpatrick J. (2012). Evaluating training programs. Berrett-Koehler. San Francisco.

McGaghie W, Issenberg S, Petrusa E \& Scalese R. (2010). A critical review of simulation-based medical education research : 2003 2009. Medical Education 44, 50-63.

Ministerio de Salud; Subsecretaría de Salud Pública. (2012). LEY NúM. 20.584; Regula los derechos y deberes que tienen las personas en relación con acciones vinculadas a atención en salud. Accedido en: https://www.leychile.cl/Navegar?idNorma=1039348\&idVersion=2012-10-01 el 10 de Junio de 2019.

Ministerio de Salud; Subsecretaría de Redes Asistenciales. (2017). Aprueba norma técnica administrativa que regula la relación asistencial docente y establece proceso de asignación de campo clínico docente de formación profesional y técnica de pregrado. Accedido en: https://www.minsal.cl/wp-content/uploads/2017/09/DS-N\%C2\%BA19.aprueba-NGTA-RAD.05-09-17.pdf el 10 de junio de 2019.

Utili F. (2007). Simulación en el aprendizaje, práctica y certificación de las competencias en medicina. Ars Medica Revista de Ciencias Médicas: 36(2).

Vermeulen J, Beeckman K, Turcksin R, Van Winkel L, Gucciardo L, Laubach M, Peersman W \& Swinnen E. (2017). The experiences of last-year student midwives with High-Fidelity Perinatal Simulation training: A qualitative descriptive study. Women and Birt, 1-9.

Villagrán I, Tejos R, Chahuan J, Uslar T, Pizarro M, Varas J, Achurra P, Leiva I, Nazar C, Sirhan M, Uribe J, Ruz C, Villafranca C, Soza R, Solís N, Fuentes-López E, Padilla O, Corvetto M \& Riquelme A. (2018). Percepción de estudiantes de pregrado de Medicina de talleres de simulación de procedimientos médico-quirúrgicos. Revista Médica de Chile 146, 786-795.

Wald H, George P, Reis S \& Taylor J. (2014). Electronic health record training in undergraduate medical education: Bridging theory to practice with curricula for empowering patient- and relationshipcentered care in the computerized setting. Academic Medicine 89, 380-386.

Weller J, Nestel D, Marshall S, Brooks P \& Conn J. (2012). Simulation in clinical teaching and learning. The Medical Journal of Australia 196, 1-5.

World Confederation for Physical Therapy. (2011). Guideline for physical therapy records management: record keeping, storage, retrieval and disposal. Accedido en: https://www.wcpt.org/sites/ wcpt.org/files/files/Guideline_records_management_complete. pdf el (12 de junio de 2019). 